Supporting Information

\title{
Synthesis and Physical Properties of a Phenanthrene-Based $[6,6]$ Hollow Bilayer Cylindrical Nanoring
}

Pingsen Huang, ${ }^{\dagger a}$ Guilin Zhuang, ${ }^{\mathrm{b}}$ Shengda Wang, ${ }^{\mathrm{a}}$ Xinyu Zhang, ${ }^{\mathrm{a}}$ Pingwu Du*a

${ }^{a}$ Hefei National Laboratory for Physical Sciences at the Microscale, iChEM, CAS Key

Laboratory of Materials for Energy Conversion, Department of Materials Science and Engineering, University of Science and Technology of China, 96 Jinzhai Road, Hefei, Anhui Province, 230026, China

${ }^{\mathrm{b}}$ College of Chemical Engineering, Zhejiang University of Technology, 18 Chaowang Road, Hangzhou, Zhejiang Province, 310032, China

+ These authors contributed equally to this work

E-mail: dupingwu@ustc.edu.cn

Tel/Fax: 86-551-63606207 


\section{Materials and General Information}

All solvents for syntheses were obtained from commercial suppliers (Innochem or Acros) and used without further purification. Air-sensitive reactions were all carried out under argon. Flash column chromatography was performed with 200-300 mesh silica gel using eluents as specified. NMR spectra were recorded on Bruker BioSpin $\left({ }^{1} \mathrm{H} 400 \mathrm{MHz},{ }^{13} \mathrm{C} 100 \mathrm{MHz}\right)$ spectrometer, and chemical shifts were reported as the delta scale in ppm relative to $\mathrm{CDCl}_{3}(\delta=7.26 \mathrm{ppm})$ for ${ }^{1} \mathrm{H} \mathrm{NMR}$ and $\mathrm{CDCl}_{3}(\delta=77.16$ ppm) for ${ }^{13} \mathrm{C}$ NMR. Data are reported as follows: chemical shift, multiplicity ( $\mathrm{s}=$ singlet, $\mathrm{d}=$ doublet, $\mathrm{t}=$ triplet, $\mathrm{m}=$ multiplet, $\mathrm{br}=$ broad signal $)$, coupling constant $(\mathrm{Hz})$, and integration. High-resolution mass spectrometry (HR-MS) analyses were carried out using MALDI-TOF-MS techniques (matrix, DCTB). UV-Vis absorption spectra were performed on a UNIC-3802 spectrophotometer. VT-NMR spectra were recorded using a JEOL JNM-ECZR spectrometer $\left({ }^{1} \mathrm{H} 600 \mathrm{MHz}\right)$. 


\section{Synthesis procedures}

Synthesis of compound 1 and other precursors. All precursors including trimeric macrocycle were synthesized according to the reported method in the literature. ${ }^{\mathrm{S} 1}$

\section{Synthesis of 3,4-bis(3-bromophenyl)-2,5-dimethylcyclopenta-2,4-dienone (2)}

To a suspension of pentan-3-one (232 mg, $2.7 \mathrm{mmol})$ and 1,2-bis(3bromophenyl)ethane-1,2-dione $\mathrm{S}^{\mathrm{3}}(512 \mathrm{mg}, 1.4 \mathrm{mmol})$ in $\mathrm{EtOH}(4.0 \mathrm{~mL})$ was added $\mathrm{KOH}$ (63 mg, $1.12 \mathrm{mmol}$ ), and the mixture was stirred at room temperature for 2 days. After the reaction was finished, the mixture was diluted by adding water, which was then extracted with EtOAc $(20 \mathrm{~mL} \times 3)$. The combined organic extract was dried over $\mathrm{Na}_{2} \mathrm{SO}_{4}$, and filtered. The solvent was evaporated and the residue was dissolved in $\mathrm{Ac}_{2} \mathrm{O}(2 \mathrm{~mL})$, and one drop of concentrated $\mathrm{H}_{2} \mathrm{SO}_{4}$ was added. The mixture was stirred at room temperature for 1 hour. Water $(20 \mathrm{~mL})$ was added to dilute the reaction and the dark yellow precipitate was collected by filtration. The crude product was purified by column chromatography (silica gel, $\mathrm{PE} / \mathrm{CH}_{2} \mathrm{Cl}_{2}=2 / 1, v / v$ ) to afford $\mathbf{2}$ as a dark yellow solid (339 mg, 58\%). Mp: 155-157 ${ }^{\circ} \mathrm{C} .{ }^{1} \mathrm{H}$ NMR (400 MHz, $\left.\mathrm{CDCl}_{3}\right): \delta 7.43(\mathrm{~s}, 2 \mathrm{H})$, 7.32-7.26 (m, 4H), $7.25(\mathrm{~s}, 2 \mathrm{H}), 7.12-7.00(\mathrm{~m}, 5 \mathrm{H}), 6.96(\mathrm{t}, J=7.9 \mathrm{~Hz}, 3 \mathrm{H}), 6.87(\mathrm{~s}$, 1H), $6.81(\mathrm{~s}, 1 \mathrm{H}), 6.68(\mathrm{~s}, 1 \mathrm{H}), 2.25(\mathrm{~s}, 4 \mathrm{H}), 1.61(\mathrm{~s}, 4 \mathrm{H}), 1.26(\mathrm{~s}, 4 \mathrm{H}), 0.61(\mathrm{~s}, 4 \mathrm{H})$.

\section{Synthesis of compound 3}

A 150-mL flask containing compound 1 (100 mg, $0.098 \mathrm{mmol})$ and 3,4-bis(3bromophenyl)-2,5-dimethylcyclopenta-2,4-dienone 2 (368 $\mathrm{mg}, 0.88 \mathrm{mmol}$ ) was evacuated and refilled with argon for 3 cycles, and then diphenylether $(3 \mathrm{~mL})$ was transferred to the flask via syringe under argon at room temperature. The mixture was 
then stirred at $260^{\circ} \mathrm{C}$ (sand bath) for 48 hours. After cooling down to room temperature, methanol $(100 \mathrm{~mL})$ was added and the precipitate was collected with filtration. The residue was purified initially by silica gel column chromatography $\left(\mathrm{PE} / \mathrm{CH}_{2} \mathrm{Cl}_{2}=3 / 1\right.$, $v / v)$ to afford the product 3 as a pale yellow solid (70 mg, 30\%). Mp: $206-208^{\circ} \mathrm{C}$. IR (KBr): 2956, 2926, 2861, 1609, 1559, 1436, 1317, 1233, 1174, 1123, 1067, 995, 892, 832, 782, $674 \mathrm{~cm}^{-1} .{ }^{1} \mathrm{H}$ NMR $\left(400 \mathrm{MHz}, \mathrm{CDCl}_{3}\right): \delta 8.23-8.19(\mathrm{~m}, 6 \mathrm{H}), 8.02-7.98(\mathrm{~m}$, 6H), $7.56(\mathrm{~s}, 6 \mathrm{H}), 7.27-6.99(\mathrm{~m}, 24 \mathrm{H}), 4.09-3.93(\mathrm{~m}, 12 \mathrm{H}), 1.88-1.76(\mathrm{~m}, 12 \mathrm{H}), 1.74-$ $1.61(\mathrm{~m}, 18 \mathrm{H}), 1.50(\mathrm{~s}, 12 \mathrm{H}), 1.56-1.48(\mathrm{~m}, 24 \mathrm{H}), 1.36-1.33(\mathrm{~m}, 24 \mathrm{H}), 0.92(\mathrm{t}, J=6.4$ $\mathrm{Hz}, 18 \mathrm{H}) .{ }^{13} \mathrm{C} \mathrm{NMR}\left(100 \mathrm{MHz}, \mathrm{CDCl}_{3}\right): \delta 143.5-142.9,141.8,139.5,138.1,133.1$, $132.3,129.8,129.5,128.9,128.2,121.4,73.7,31.9,30.6,26.0,22.8,19.8,14.2$. HRMS (MALDI-TOF-MS) $m / z$ calcd. for $\mathrm{C}_{138} \mathrm{H}_{138} \mathrm{Br}_{6} \mathrm{O}_{6}[M]^{+}$: 2371.5566, found 2371.5515. UV-Vis: $\lambda_{\max }=261 \mathrm{~nm}$.

\section{Synthesis of HBCNR}

Bis(cyclooctadiene)nickel(0) (66mg, $0.24 \mathrm{mmol})$, cyclooctadiene $(0.2 \mathrm{~mL})$, and 2,2'bipyridine (36.4 mg, $0.24 \mathrm{mmol}$ ) were dissolved in DMF (5 mL), and stirred for 1hour at $80{ }^{\circ} \mathrm{C}$ (oil bath) under absence of light for activation of nickel catalyst. A solution of $3(15 \mathrm{mg}, 0.006 \mathrm{mmol})$ in dry toluene $(5 \mathrm{~mL})$ was quickly added to the reaction mixture and stirred for 24 hours at same temperature. After cooling to ambient temperature, the reaction was quenched by saturated aqueous solution of $\mathrm{NH}_{4} \mathrm{Cl}$. After extraction of organic layer with dichloromethane washing with brine, the organic layer was separated and dried over $\mathrm{MgSO}_{4}$. After removing solvent in vacuo, the crude material was 
subjected to flash column chromatography on silica gel $\left(\mathrm{PE} / \mathrm{CH}_{2} \mathrm{Cl}_{2}=2.5 / 1, v / v\right)$ to afford the target compound $(4.5 \mathrm{mg}, 38 \%)$ as white solid. Mp: $243-245{ }^{\circ} \mathrm{C}$. IR (KBr): 2956, 2926, 2856, 1600, 1461, 1439, 1386, 1317, 1261, 1171, 1121, 1060, 889, 801, $715 \mathrm{~cm}^{-1} .{ }^{1} \mathrm{H}$ NMR $\left(400 \mathrm{MHz}, \mathrm{CDCl}_{3}\right): \delta 8.34(\mathrm{~s}, 6 \mathrm{H}), 7.98(\mathrm{~d}, J=8.3 \mathrm{~Hz}, 6 \mathrm{H}), 7.88-$ $7.78(\mathrm{~m}, 12 \mathrm{H}), 7.59(\mathrm{~d}, J=8.2 \mathrm{~Hz}, 6 \mathrm{H}), 7.50(\mathrm{~d}, J=7.8 \mathrm{~Hz}, 6 \mathrm{H}), 7.33(\mathrm{t}, J=7.8 \mathrm{~Hz}$, $6 \mathrm{H}), 4.02(\mathrm{dd}, J=13.8,6.9 \mathrm{~Hz}, 12 \mathrm{H}), 1.86-1.78(\mathrm{~m}, 12 \mathrm{H}), 1.73(\mathrm{~s}, 18 \mathrm{H}), 1.52-1.47(\mathrm{~m}$, $12 \mathrm{H}), 1.39-1.30(\mathrm{~m}, 24 \mathrm{H}), 0.91(\mathrm{t}, J=6.6 \mathrm{~Hz}, 18 \mathrm{H}) .{ }^{13} \mathrm{C} \mathrm{NMR}\left(100 \mathrm{MHz}, \mathrm{CDCl}_{3}\right): \delta$ $143.3,142.0,140.9,137.5,134.7,129.9,129.3,128.8,128.1,127.7,127.3,123.2,121.1$ 73.7, 31.9, 30.6, 26.0, 22.9, 22.8, 14.2. HR-MS (MALDI-TOF-MS) $\mathrm{m} / \mathrm{z}$ calcd. for $\mathrm{C}_{138} \mathrm{H}_{138} \mathrm{O}_{6}[M]^{+}:$1892.0527, found 1892.0509 . UV-Vis: $\lambda_{\max }=260 \mathrm{~nm}$.

Computational detail. Density function theory calculation was carried out using Gaussian 09 software $^{\mathrm{S} 4}$ at the theoretical level of B3LYP/6-31G(d, p), where DFTD3(BJ) methodologies can be used for dispersion correction. ${ }^{\mathrm{S} 5}$ The solvent effect was treated by the Polarizable continuum model (PCM) method. ${ }^{\mathrm{S} 6}$ The relaxed ground-state structure was further validated by frequency analysis. Moreover, time-dependent density functional theory (TD-DFT) ${ }^{\mathrm{S} 7}$ with $\mathrm{PBE} 0 / 6-31 \mathrm{G}(\mathrm{d}, \mathrm{p}) / / \mathrm{PCM}$ was used to simulate the UV spectra. The convergence for SCF calculation was set to $1 \times 10^{-8}$ a.u.. In the initial structures, hydrocarbon substituent groups were replaced with hydrogen atoms for the reduction of computational cost. The tension energy was calculated using the computational methods reported by K. Itami. ${ }^{\text {S7-S8 }}$ 


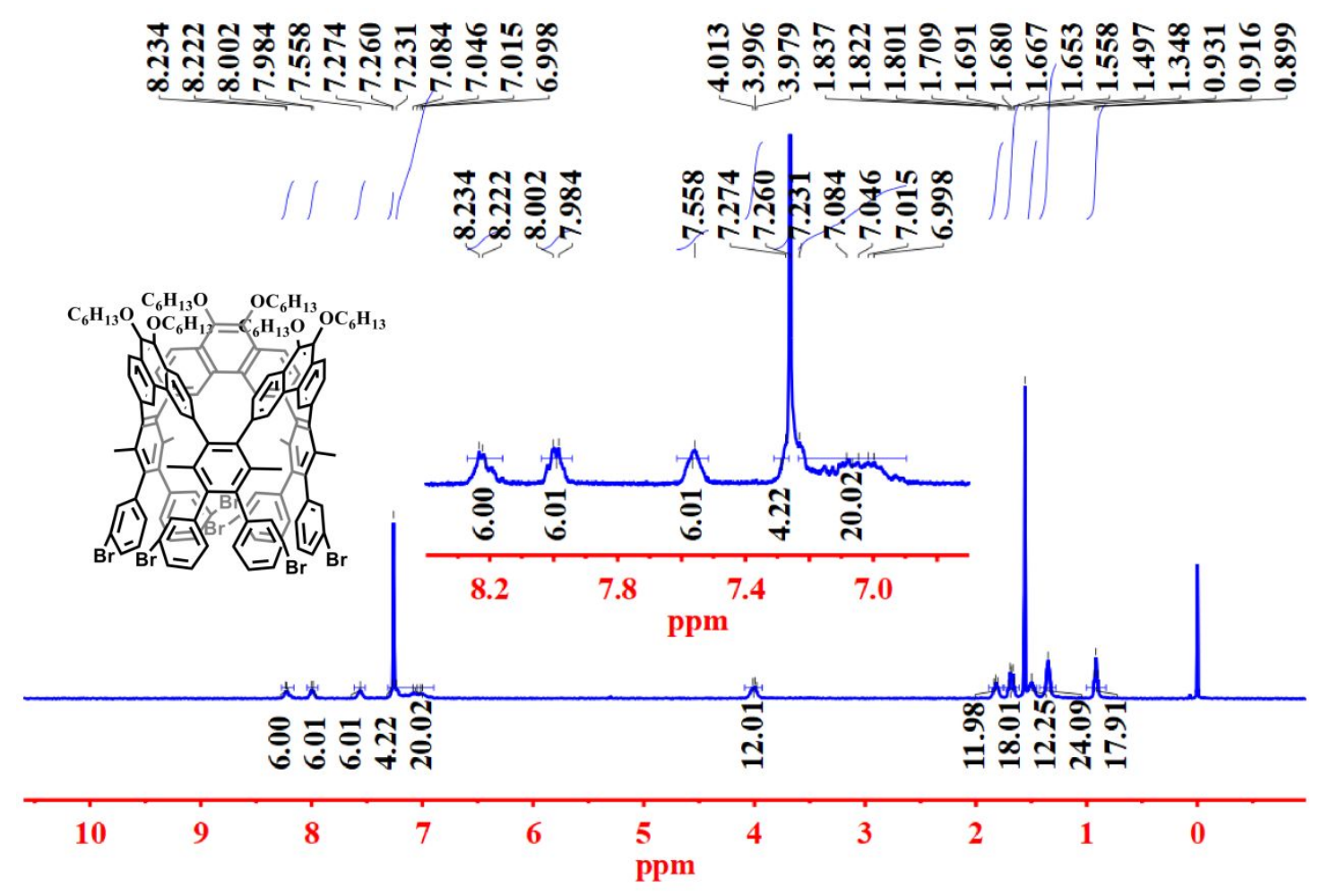

Figure S1. ${ }^{1} \mathrm{H}$ NMR spectrum $(400 \mathrm{MHz})$ of compound 3 in $\mathrm{CDCl}_{3}$. 


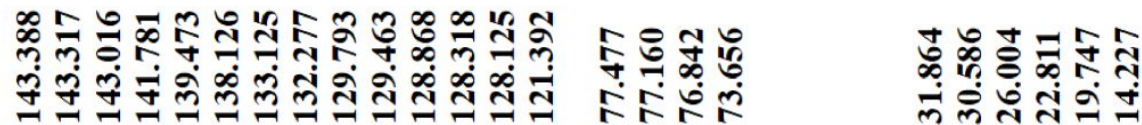

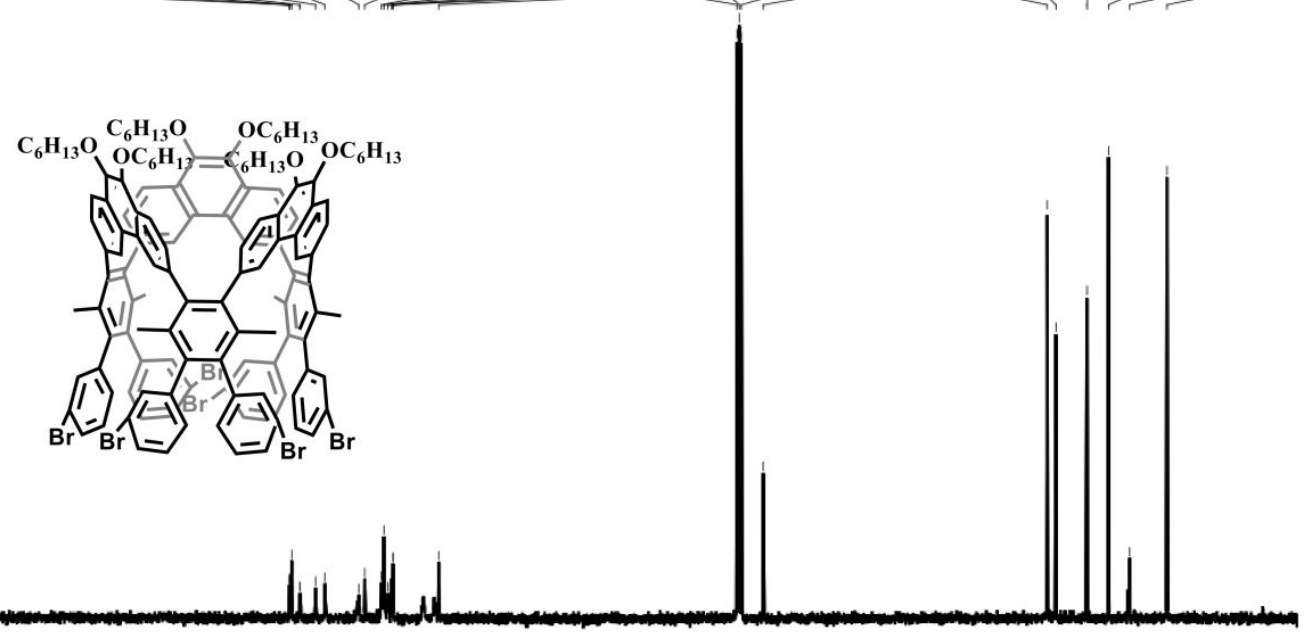

\begin{tabular}{llllllllll}
\hline 200 & 180 & 160 & 140 & 120 & $\begin{array}{c}100 \\
\text { ppm }\end{array}$ & 80 & 60 & 40 & 20
\end{tabular}

Figure S2. ${ }^{13} \mathrm{C}$ NMR spectrum (100 MHz) of compound 3 in $\mathrm{CDCl}_{3}$. 


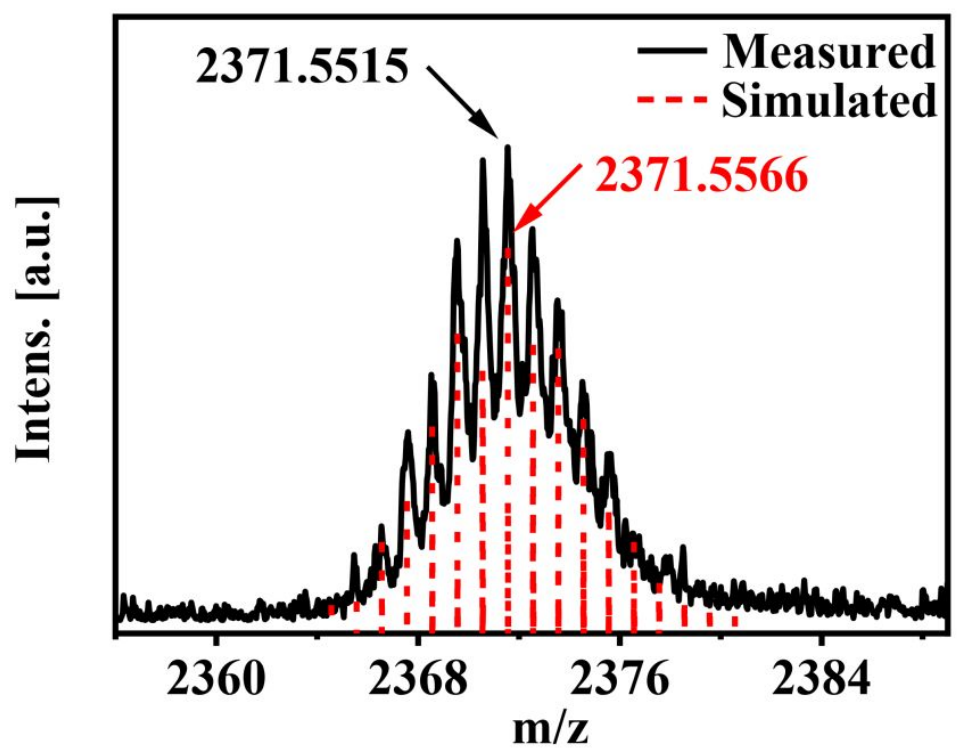

Figure S3. MALDI-TOF-MS and simulated data for compound 3. 


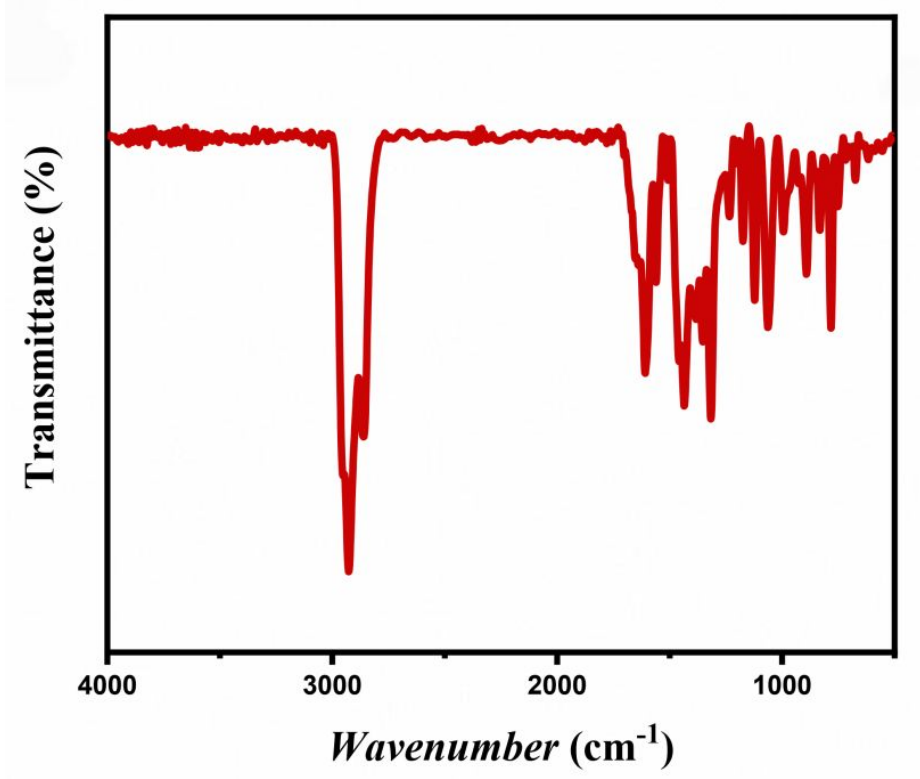

Figure S4. FTIR spectrum of compound 3. 


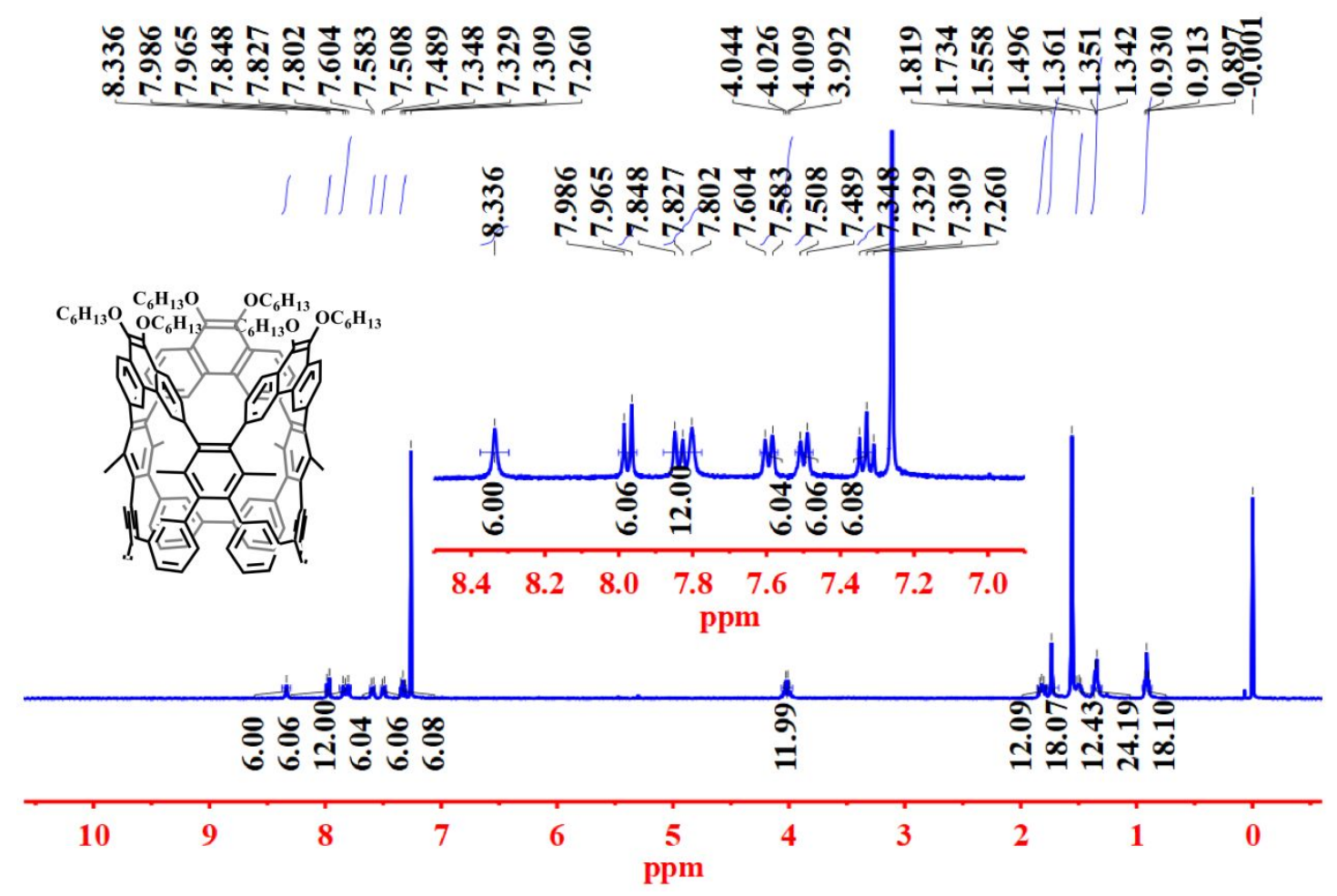

Figure S5. ${ }^{1} \mathrm{H}$ NMR spectrum (400 MHz) of $\mathbf{H B C N R}$ in $\mathrm{CDCl}_{3}$. 


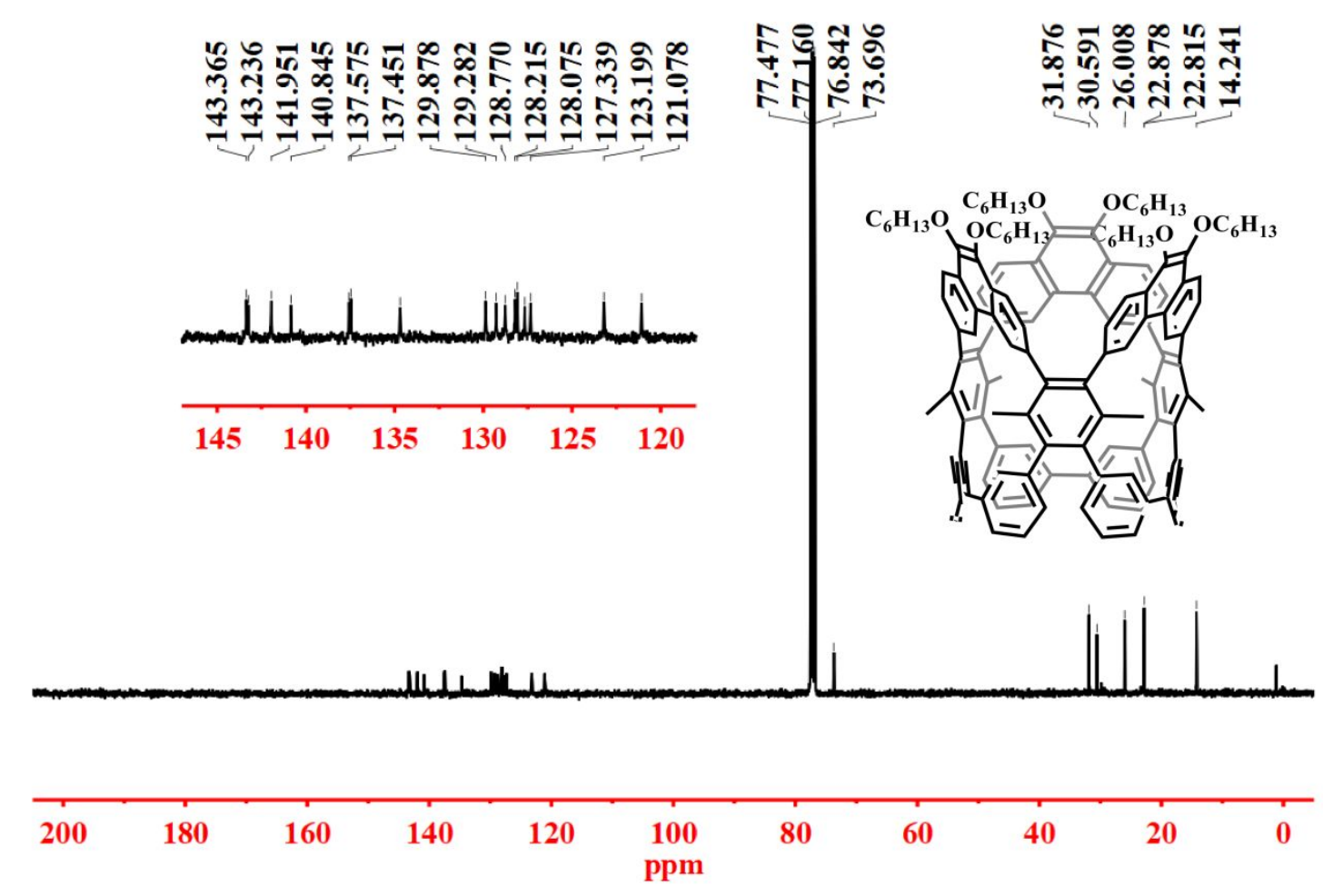

Figure S6. ${ }^{13} \mathrm{C}$ NMR spectrum $(100 \mathrm{MHz})$ of $\mathbf{H B C N R}$ in $\mathrm{CDCl}_{3}$. 


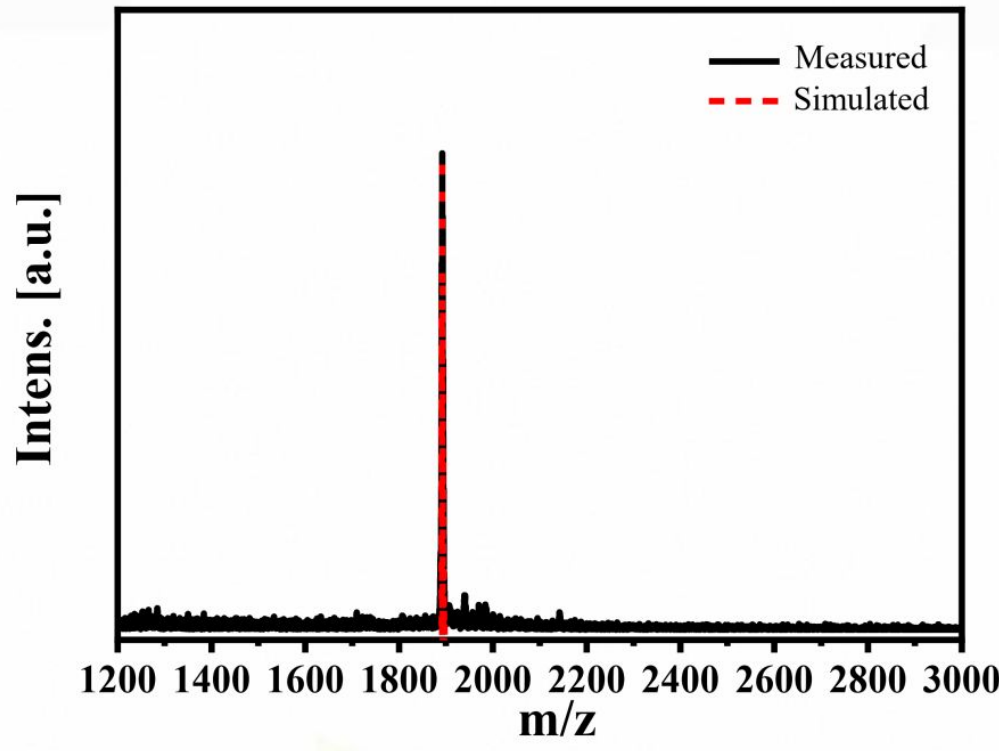

Figure S7. MALDI-TOF-MS and simulated data for HBCNR. 


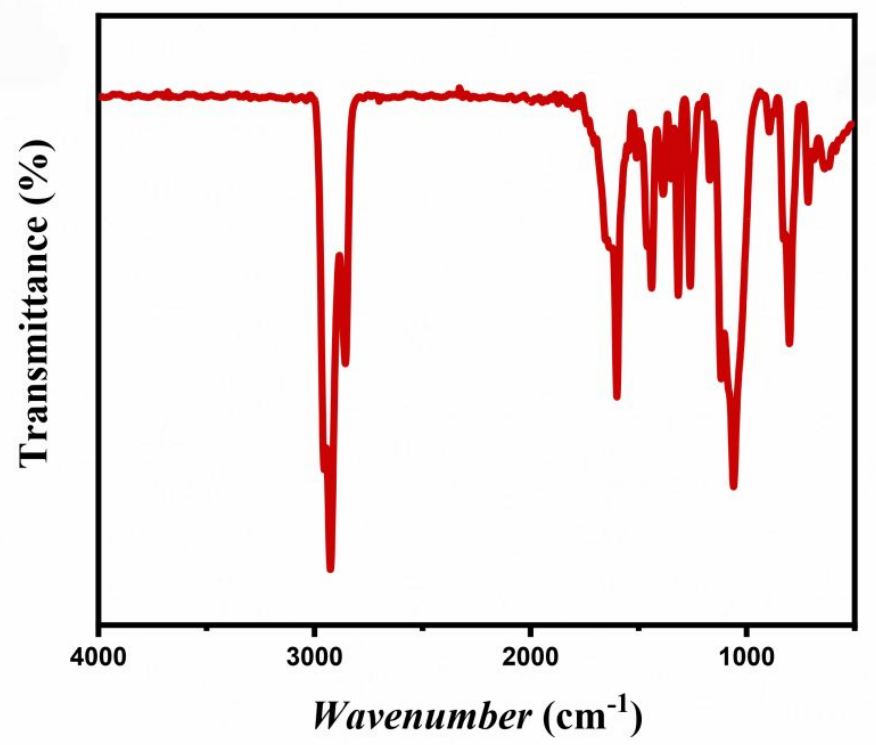

Figure S8. FTIR spectrum of HBCNR. 
(c) $\mathrm{CH} \uparrow$

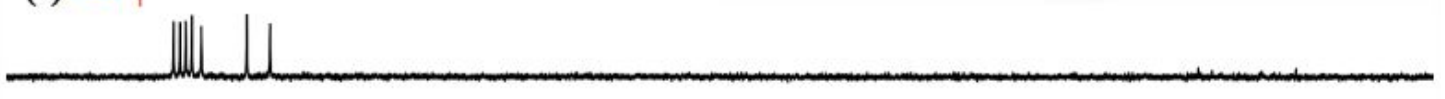

(b) $\mathrm{CH} \mathrm{CH}_{3} \uparrow \mathrm{CH}_{2} \downarrow$

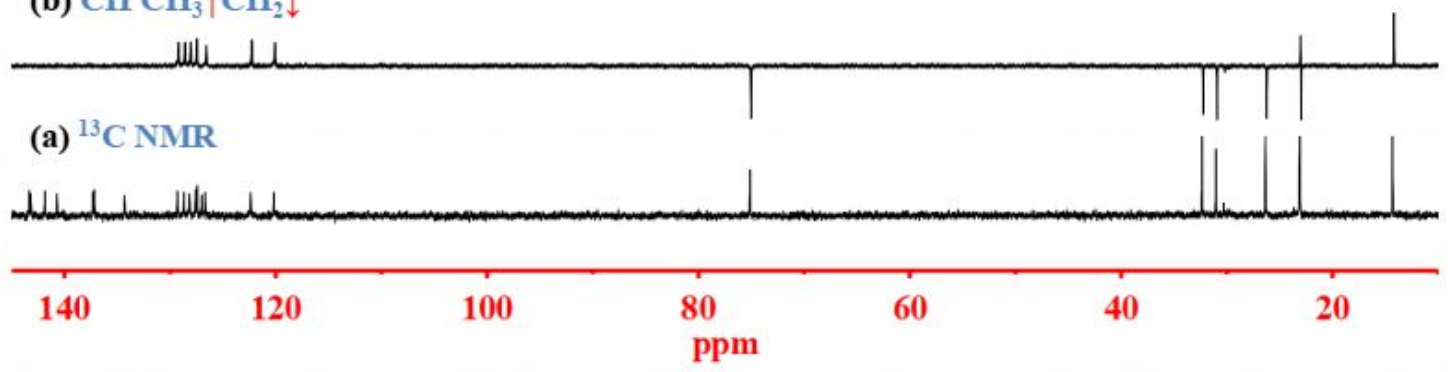

Figure S9. (a) ${ }^{13} \mathrm{C}$ NMR, (b) DEPT- $135^{\circ} \mathrm{NMR}$, and (c) DEPT- $90^{\circ} \mathrm{NMR}$ spectra (400 $\mathrm{MHz}$ ) of $\mathbf{H B C N R}$ in $\mathrm{CDCl}_{3}$. 


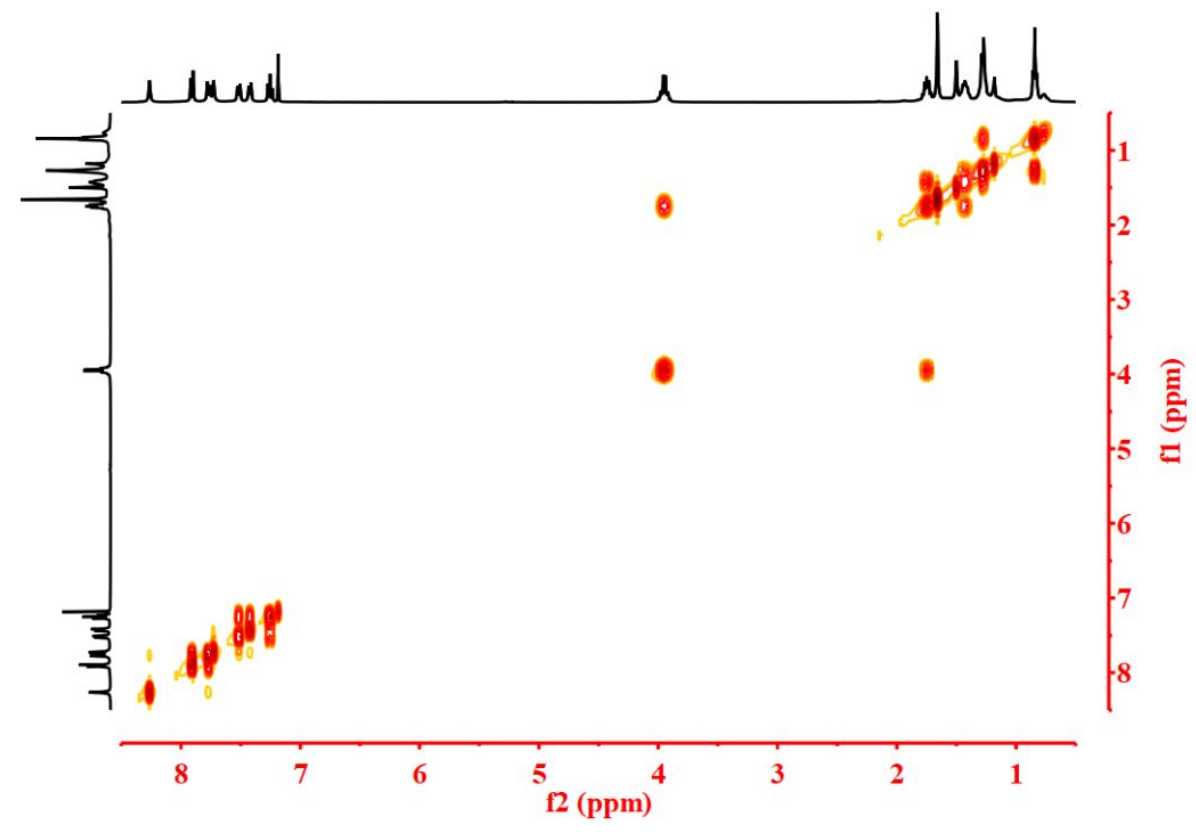

Figure S10. The expanded 2D ${ }^{1} \mathrm{H}-{ }^{1} \mathrm{H}$ COSY NMR spectrum (400 MHz) of HBCNR in $\mathrm{CDCl}_{3}$.

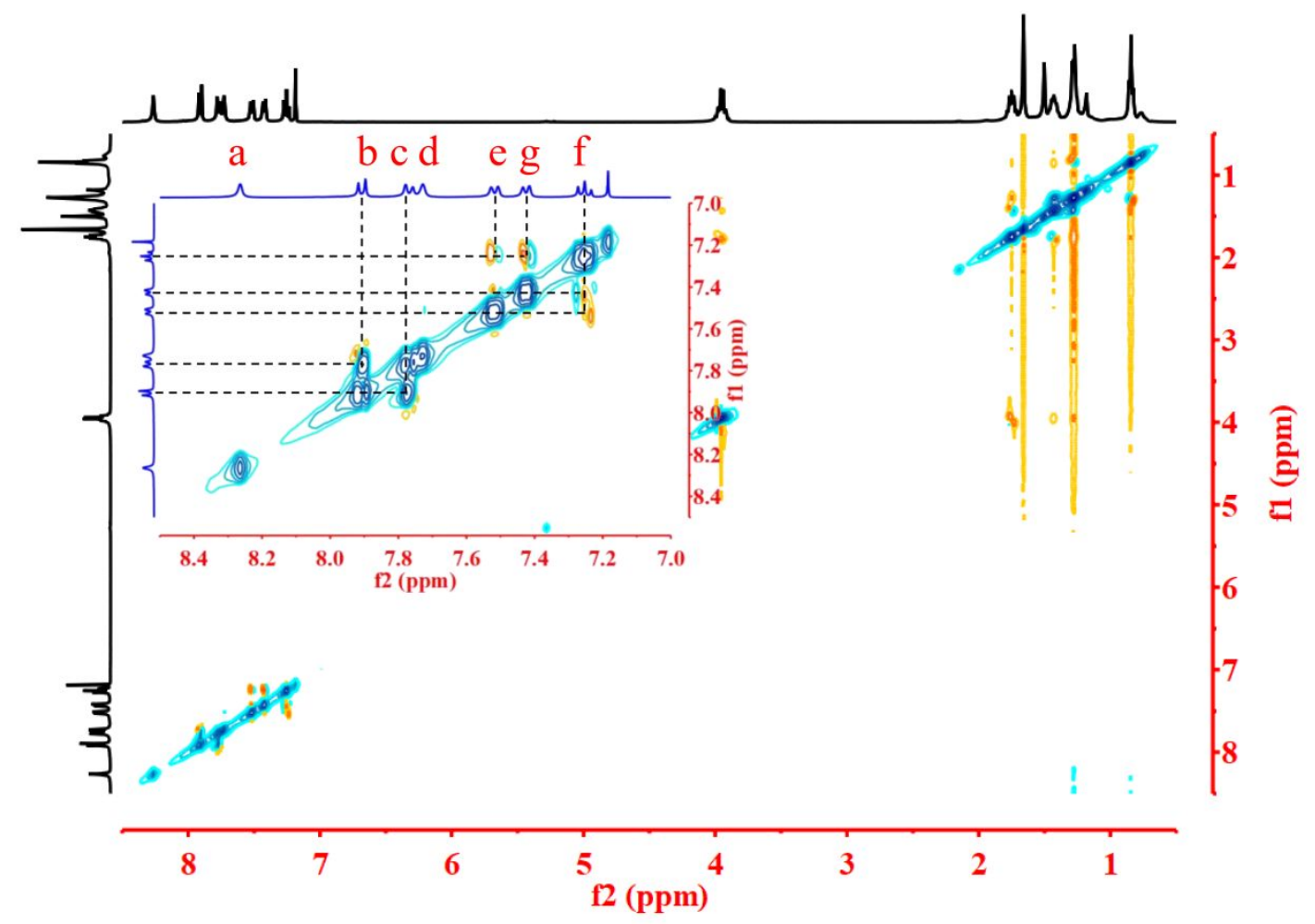

Figure S11. The expanded 2D ${ }^{1} \mathrm{H}-{ }^{1} \mathrm{H}$ ROESY NMR spectrum (400 MHz) of HBCNR in $\mathrm{CDCl}_{3}$. 


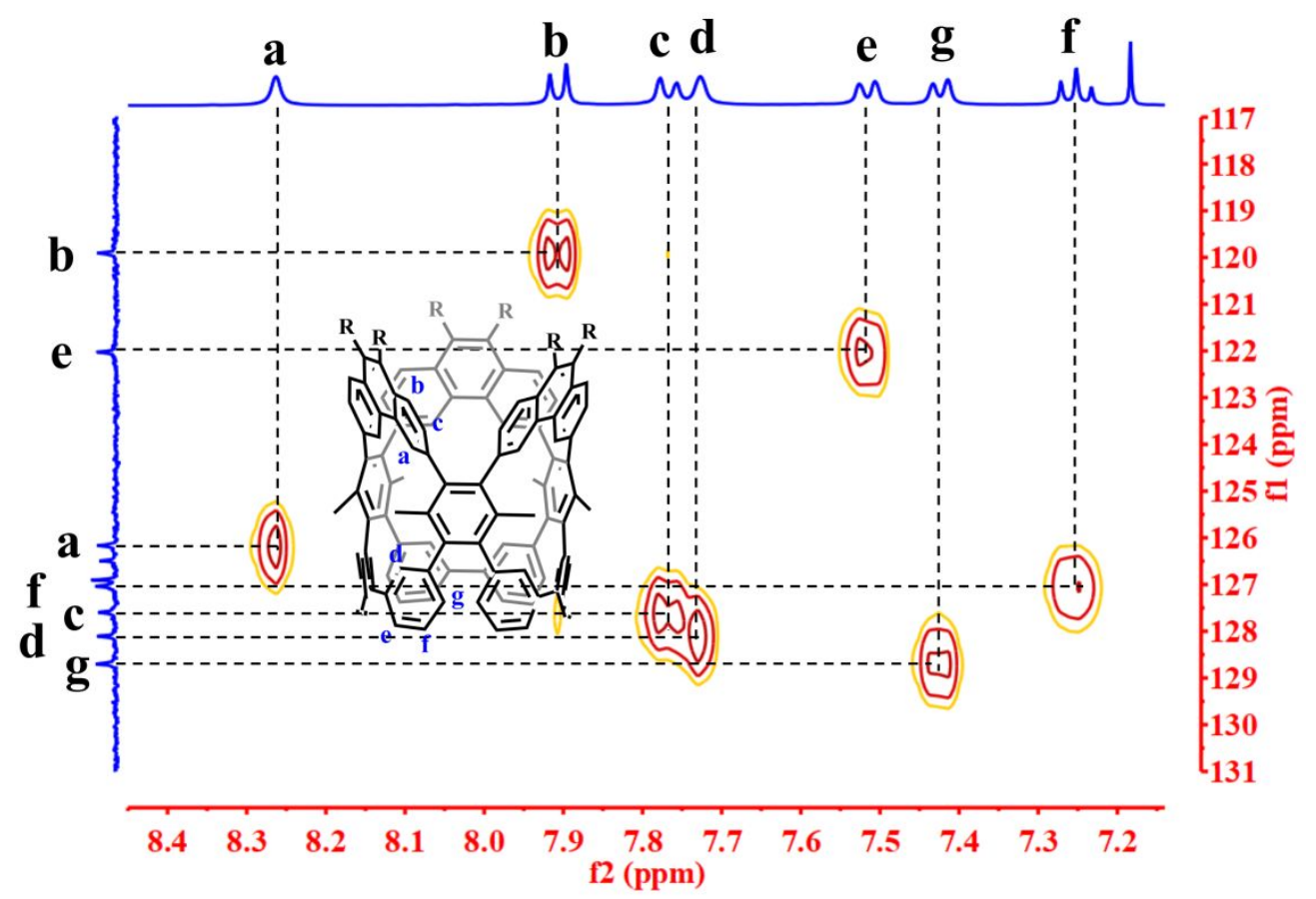

Figure S12. The expanded 2D (H, C)-HSQC NMR spectrum (400 MHz) of HBCNR in $\mathrm{CDCl}_{3}$. 

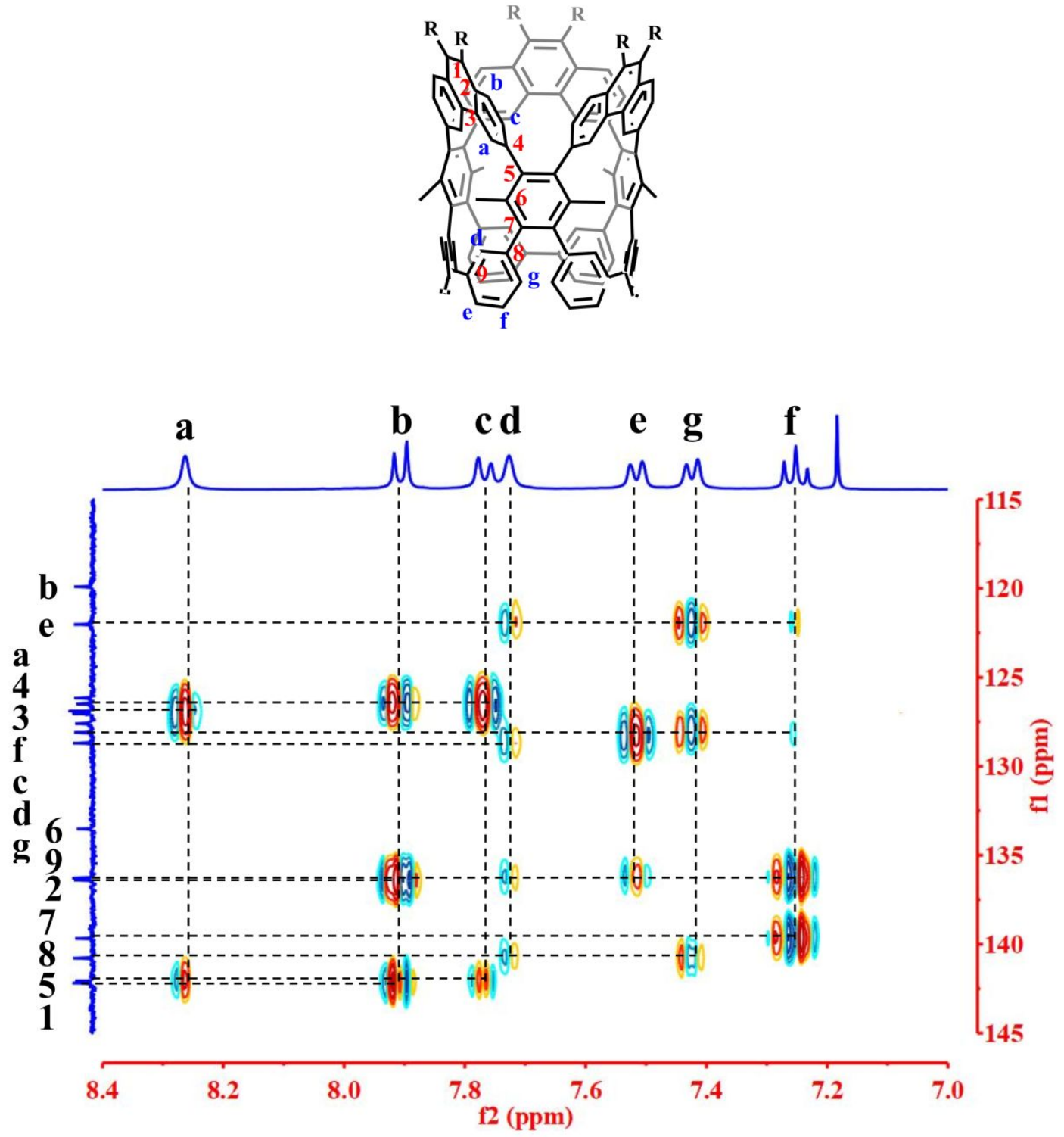

Figure S13. The expanded 2D (H, C)-HMBC NMR spectrum (400 MHz) of HBCNR in $\mathrm{CDCl}_{3}$. 
(a)

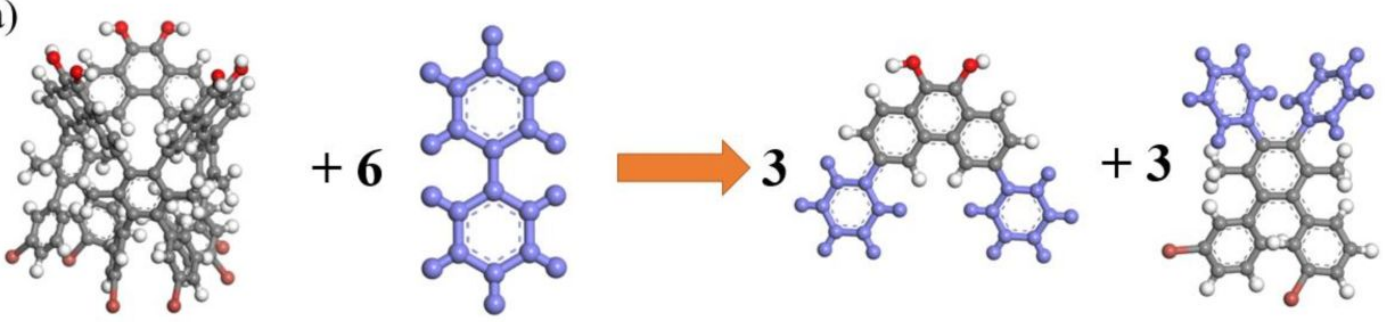

(b)

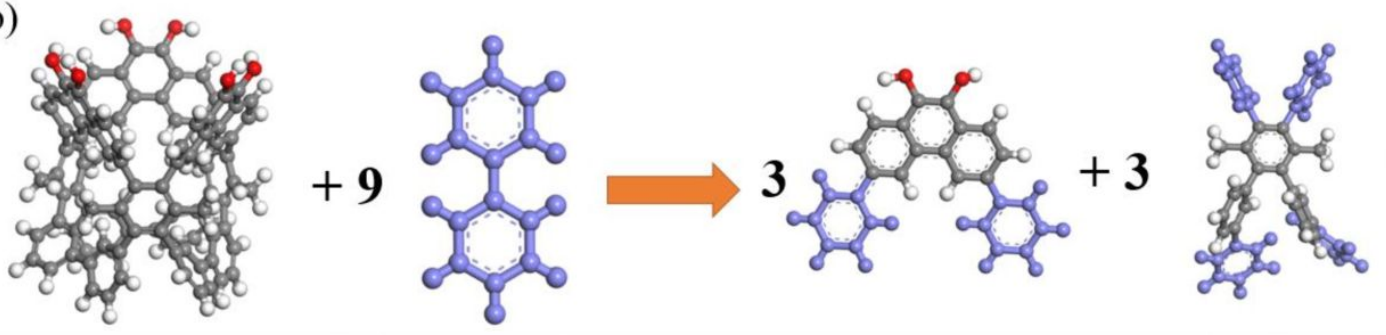

Figure S14. Homodesmotic equation for the calculation of strain energy for compound 3 (a) and HBCNR (b). 
(a)

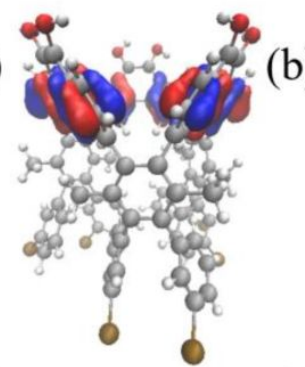

(e)

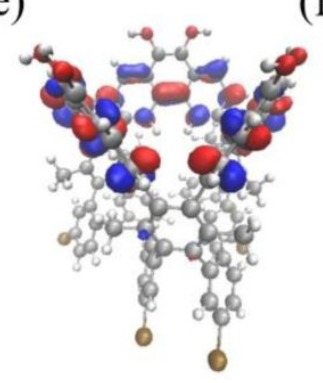

(b)

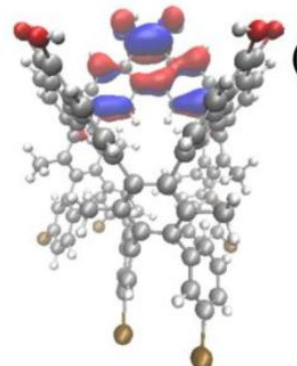

(f)

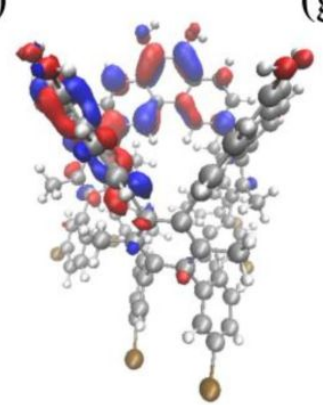

(c)

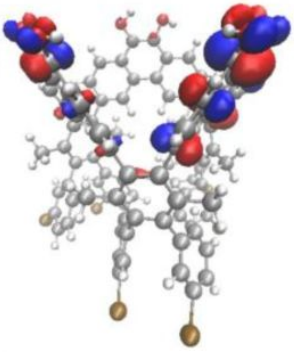

(g)

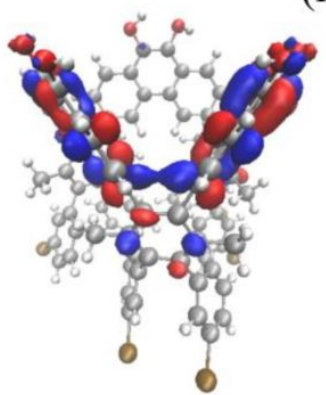

(d)

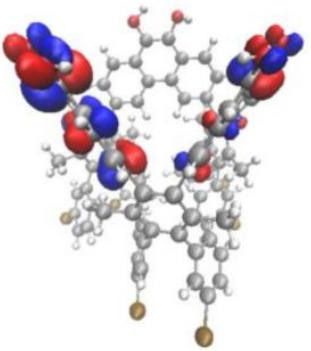

(h)

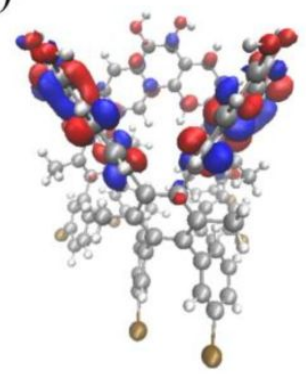

Figure S15. Frontier molecular orbits of compound 3: HOMO-3 (a), HOMO-2 (b), HOMO-1 (c), HOMO (d), LUMO (e), LUMO+1 (f), LUMO+2 (g) and LUMO+3 (h). 


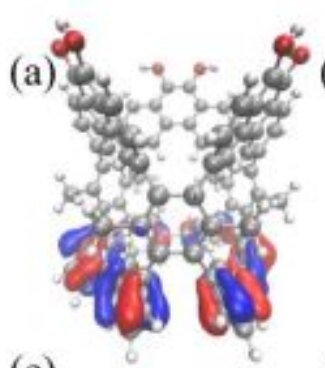

(e)

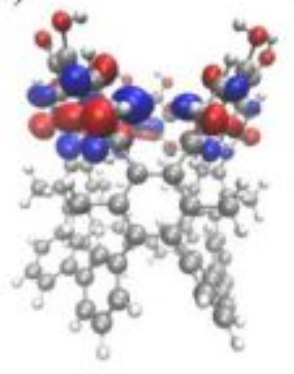

(b)

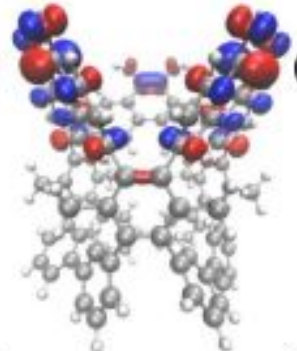

(f)

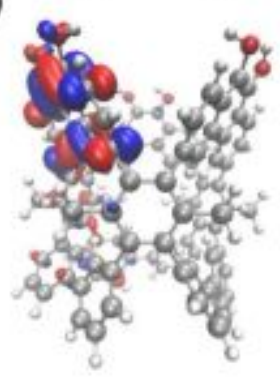

(c)

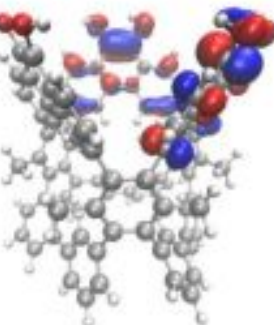

(g)

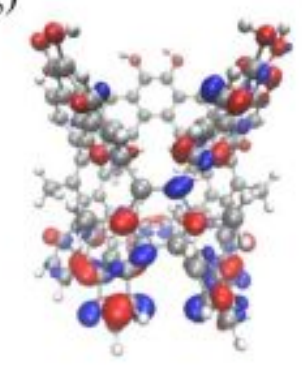

(d)

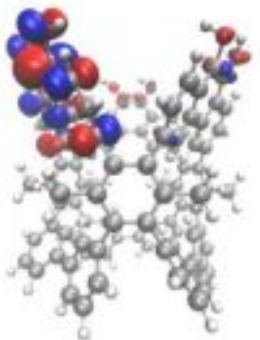

(h)

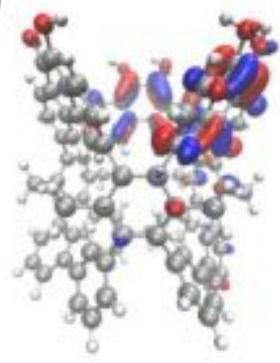

Figure S16. Frontier molecular orbits of HBCNR: HOMO-3 (a), HOMO-2 (b), HOMO-1 (c), HOMO (d), LUMO (e), LUMO+1 (f), LUMO+2 (g) and LUMO+3 (h). 
Table S1. Energy level of frontier molecular orbitals $(\mathrm{eV})$

\begin{tabular}{ccc}
\hline Label & Compound 3 & HBCNR \\
\hline LUMO+3 & -1.186 & -1.037 \\
LUMO+2 & -1.352 & -1.078 \\
LUMO+1 & -1.360 & -1.091 \\
LUMO & -1.496 & -1.224 \\
HOMO & -5.302 & -5.147 \\
HOMO-1 & -5.348 & -5.183 \\
HOMO-2 & -5.466 & -5.215 \\
HOMO-3 & -6.156 & -5.800 \\
DE(HOMO-LUMO) & 3.806 & 3.923 \\
\hline
\end{tabular}


Table S2. Oscillator strengths and transitions of the peaks from experiment and DFT calculations under the theoretical level of PBE0/6-31G(d,p)//PCM)

\begin{tabular}{|c|c|c|c|}
\hline$\lambda_{\text {Exp. }}$ & $\lambda_{\mathrm{DFT}}$ & $f_{\text {osc }}$ & Transitions \\
\hline \multicolumn{4}{|c|}{ Compound 3} \\
\hline \multirow{2}{*}{311} & 347 & 0.0838 & $\begin{array}{l}\text { HOMO-2 } \rightarrow \text { LUMO }(53 \%) \\
\text { HOMO- } 1 \rightarrow \text { LUMO }+5(22 \%)\end{array}$ \\
\hline & 331 & 0.3694 & $\begin{array}{l}\mathrm{HOMO} \rightarrow \text { LUMO+1 }(46 \%) \\
\text { HOMO-1 } \rightarrow \text { LUMO+2 }(27 \%)\end{array}$ \\
\hline \multirow[t]{2}{*}{259} & 289 & 0.0559 & $\begin{array}{l}\text { HOMO-2 } \rightarrow \text { LUMO+6 (47\%) } \\
\text { HOMO-2 } \rightarrow \text { LUMO+7 (28\%) }\end{array}$ \\
\hline & 270 & 0.0656 & HOMO-2 $\rightarrow$ LUMO+10 (46\%) \\
\hline \multicolumn{4}{|c|}{ HBCNR } \\
\hline \multirow{2}{*}{306} & 343 & 0.0912 & $\begin{array}{l}\text { HOMO-2 } \rightarrow \text { LUMO }(53 \%) \\
\text { HOMO-1 } \rightarrow \text { LUMO+5 }(22 \%)\end{array}$ \\
\hline & 322 & 0.3232 & $\begin{array}{l}\text { HOMO-2 } \rightarrow \text { LUMO+3 }(42 \%) \\
\text { HOMO-1 } \rightarrow \text { LUMO+2 (19\%) }\end{array}$ \\
\hline \multirow{2}{*}{261} & 270 & 0.0401 & HOMO-6 $\rightarrow$ LUMO (33\%) \\
\hline & 269 & 0.0302 & HOMO-6 $\rightarrow$ LUMO (34\%) \\
\hline
\end{tabular}


Table S3. Energy (a.u.) of the compound 3 and HBCNR, and other involved moieties at theoretical level of D3(BJ)-B3LYP/6-31G(d,p)

\begin{tabular}{|c|c|c|c|}
\hline \multicolumn{2}{|c|}{ Compound 3} & \multicolumn{2}{|c|}{ HBCNR } \\
\hline Compound 3 & -19808.9733408 & HBCNB & -4378.7297265 \\
\hline Biphenyl & -463.362583126 & Biphenyl & -463.362583126 \\
\hline Fragment-2OH & -1152.2242836 & Fragment-2OH & -1152.2242836 \\
\hline Fragment-2Br & -6377.4953257 & Fragment & -1697.4650376 \\
\hline $\begin{array}{l}\text { Strain energy } \\
(\mathrm{kcal} / \mathrm{mol})\end{array}$ & 6.3 & $\begin{array}{c}\text { Strain energy } \\
(\mathrm{kcal} / \mathrm{mol})\end{array}$ & 47.1 \\
\hline
\end{tabular}


Table S4. Relaxed-structure coordinate of compound 3 and HBCNR

\begin{tabular}{|c|c|c|c|c|c|c|c|}
\hline \multicolumn{8}{|c|}{ Compound $\mathbf{3}$} \\
\hline $\mathrm{C}$ & 3.093906 & 3.504104 & -3.249202 & $\mathrm{O}$ & -7.675884 & 3.774406 & 3.980956 \\
\hline $\mathrm{C}$ & 4.357323 & 2.577928 & 1.484021 & $\mathrm{O}$ & -7.778800 & 1.446631 & 5.273827 \\
\hline $\mathrm{C}$ & 4.344793 & -2.619475 & 1.480653 & $\mathrm{O}$ & -7.771196 & -5.392361 & -1.295170 \\
\hline $\mathrm{C}$ & 3.623227 & -4.776723 & -3.758709 & $\mathrm{O}$ & -7.565043 & -5.447807 & 1.379583 \\
\hline $\mathrm{C}$ & 4.015330 & 4.540535 & -3.102427 & $\mathrm{O}$ & -7.794230 & 3.903099 & -3.854690 \\
\hline $\mathrm{C}$ & 4.151008 & 3.951485 & 1.381659 & $\mathrm{O}$ & -7.704437 & 1.624275 & -5.233457 \\
\hline $\mathrm{C}$ & 4.137488 & -3.223845 & 2.718144 & $\mathrm{C}$ & 2.501778 & -1.430830 & 5.667395 \\
\hline $\mathrm{C}$ & 3.967936 & -4.960334 & -2.421978 & $\mathrm{C}$ & 3.719915 & -0.819782 & 5.944099 \\
\hline $\mathrm{C}$ & 3.690403 & 5.607166 & -2.268060 & $\mathrm{C}$ & 4.029902 & 0.444513 & 5.449208 \\
\hline $\mathrm{C}$ & 2.884788 & 4.484374 & 1.160468 & $\mathrm{C}$ & 3.092102 & 1.094863 & 4.647652 \\
\hline $\mathrm{C}$ & 2.874749 & -3.279841 & 3.300457 & $\mathrm{C}$ & 1.886884 & 0.473582 & 4.322535 \\
\hline $\mathrm{C}$ & 3.064365 & -4.557875 & -1.438959 & $\mathrm{C}$ & 1.585171 & -0.799800 & 4.818725 \\
\hline $\mathrm{C}$ & 1.889919 & 3.522972 & -2.546083 & $\mathrm{C}$ & 2.830917 & -1.240422 & -4.471463 \\
\hline $\mathrm{C}$ & 3.258383 & 1.726466 & 1.352082 & $\mathrm{C}$ & 4.102243 & -0.791971 & -4.127162 \\
\hline $\mathrm{C}$ & 3.250659 & -2.053961 & 0.822354 & $\mathrm{C}$ & 4.319717 & -0.015593 & -2.991508 \\
\hline $\mathrm{C}$ & 2.403498 & -4.218237 & -4.125516 & $\mathrm{C}$ & 3.226776 & 0.306132 & -2.183863 \\
\hline $\mathrm{C}$ & 1.573797 & 4.594947 & -1.703836 & $\mathrm{C}$ & 1.956129 & -0.179630 & -2.492201 \\
\hline $\mathrm{C}$ & 1.993017 & 2.246857 & 1.081142 & $\mathrm{C}$ & 1.746918 & -0.973259 & -3.630184 \\
\hline $\mathrm{C}$ & 1.989562 & -2.062625 & 1.418079 & $\mathrm{H}$ & 3.331379 & 2.661086 & -3.888752 \\
\hline $\mathrm{C}$ & 1.522177 & -3.765889 & -3.136970 & $\mathrm{H}$ & 5.351327 & 2.184377 & 1.660036 \\
\hline $\mathrm{C}$ & 2.473270 & 5.661812 & -1.597450 & $\mathrm{H}$ & 5.336202 & -2.589286 & 1.044625 \\
\hline $\mathrm{C}$ & 1.795020 & 3.631008 & 0.964778 & $\mathrm{H}$ & 4.969943 & 4.518809 & -3.614290 \\
\hline $\mathrm{C}$ & 1.790854 & -2.660277 & 2.671929 & $\mathrm{H}$ & 4.924218 & -5.396773 & -2.159679 \\
\hline $\mathrm{C}$ & 1.858469 & -3.952908 & -1.791440 & $\mathrm{H}$ & 2.759588 & 5.556177 & 1.094036 \\
\hline $\mathrm{C}$ & 0.468201 & 4.135881 & 0.494914 & $\mathrm{H}$ & 2.748623 & -3.763380 & 4.259338 \\
\hline $\mathrm{C}$ & 0.468379 & -2.491545 & 3.349823 & $\mathrm{H}$ & 3.317542 & -4.685618 & -0.392254 \\
\hline $\mathrm{C}$ & 0.416293 & -1.627344 & -3.823365 & $\mathrm{H}$ & 1.205238 & 2.685625 & -2.625710 \\
\hline $\mathrm{C}$ & 0.348001 & 4.545924 & -0.849505 & $\mathrm{H}$ & 3.393374 & 0.654837 & 1.454080 \\
\hline $\mathrm{C}$ & 0.355370 & -1.516083 & 4.362142 & $\mathrm{H}$ & 3.386191 & -1.600017 & -0.153767 \\
\hline $\mathrm{C}$ & 0.294977 & -2.996498 & -3.507088 & $\mathrm{H}$ & 2.158489 & -4.093833 & -5.173061 \\
\hline $\mathrm{C}$ & -0.538417 & 0.562561 & -4.623296 & $\mathrm{H}$ & 1.151427 & 1.576981 & 0.932639 \\
\hline $\mathrm{C}$ & -0.917931 & 4.783282 & -1.404743 & $\mathrm{H}$ & 1.152167 & -1.580345 & 0.922787 \\
\hline $\mathrm{C}$ & -0.484806 & 3.741291 & 2.793756 & $\mathrm{H}$ & 2.243539 & 6.507634 & -0.961431 \\
\hline $\mathrm{C}$ & -0.908267 & -1.141976 & 4.842689 & $\mathrm{H}$ & 1.188709 & -3.593036 & -1.017864 \\
\hline $\mathrm{C}$ & -0.490878 & -4.304784 & 1.890325 & $\mathrm{H}$ & -0.524350 & 1.273470 & -3.788643 \\
\hline $\mathrm{C}$ & -0.971671 & -3.592139 & -3.423064 & $\mathrm{H}$ & -1.349955 & 0.867001 & -5.285835 \\
\hline $\mathrm{C}$ & -0.716751 & -0.868675 & -4.160733 & $\mathrm{H}$ & -1.294790 & 4.164928 & 3.389317 \\
\hline $\mathrm{C}$ & -1.049909 & 5.159279 & -2.863316 & $\mathrm{H}$ & 0.465088 & 4.138912 & 3.157415 \\
\hline $\mathrm{C}$ & -0.663616 & 4.056989 & 1.323227 & $\mathrm{H}$ & -0.455967 & -3.951565 & 0.852889 \\
\hline $\mathrm{C}$ & -1.033627 & -0.055220 & 5.886692 & $\mathrm{H}$ & -1.312417 & -5.019270 & 1.956507 \\
\hline $\mathrm{C}$ & -0.667191 & -3.172560 & 2.880951 & $\mathrm{H}$ & -1.066688 & 4.272159 & -3.507353 \\
\hline $\mathrm{C}$ & -1.941888 & 4.247428 & 0.752114 & $\mathrm{H}$ & -0.206241 & 5.773709 & -3.183638 \\
\hline $\mathrm{C}$ & -1.943419 & -2.760110 & 3.326014 & $\mathrm{H}$ & -1.049880 & 0.941821 & 5.431137 \\
\hline $\mathrm{C}$ & -1.995766 & -1.455016 & -4.029346 & $\mathrm{H}$ & -0.187605 & -0.080478 & 6.576276 \\
\hline $\mathrm{C}$ & -2.062047 & 4.563074 & -0.613213 & $\mathrm{H}$ & -2.567909 & 0.698218 & -2.644667 \\
\hline $\mathrm{C}$ & -2.056547 & -1.719592 & 4.266256 & $\mathrm{H}$ & -2.519435 & 1.982414 & 1.943573 \\
\hline $\mathrm{C}$ & -2.116937 & -2.793557 & -3.613318 & $\mathrm{H}$ & -2.509117 & -2.702457 & 0.767282 \\
\hline
\end{tabular}




\begin{tabular}{|c|c|c|c|c|c|c|c|}
\hline $\mathrm{C}$ & -3.349450 & 0.505521 & -3.367219 & $\mathrm{H}$ & -2.555925 & 2.400785 & -1.789538 \\
\hline $\mathrm{C}$ & -3.348374 & 4.304402 & -1.334351 & $\mathrm{H}$ & -2.531070 & 0.381719 & 2.975561 \\
\hline $\mathrm{C}$ & -3.303823 & 2.705018 & 2.123708 & $\mathrm{H}$ & -2.522920 & -2.826482 & -1.137059 \\
\hline $\mathrm{C}$ & -3.337392 & -0.955246 & 4.397439 & $\mathrm{H}$ & -4.085694 & 5.905810 & 1.361904 \\
\hline $\mathrm{C}$ & -3.299008 & -3.205037 & 1.308775 & $\mathrm{H}$ & -4.117534 & -4.041775 & 4.484675 \\
\hline $\mathrm{C}$ & -3.393942 & -3.286222 & -3.006323 & $\mathrm{H}$ & -4.129387 & -1.721485 & -5.789284 \\
\hline $\mathrm{C}$ & -3.214970 & -0.611225 & -4.175615 & $\mathrm{H}$ & -4.391659 & 6.186603 & -1.103351 \\
\hline $\mathrm{C}$ & -3.405878 & 3.055257 & -1.932201 & $\mathrm{H}$ & -4.392499 & -2.082813 & 5.914359 \\
\hline $\mathrm{C}$ & -3.165079 & 3.958948 & 1.551682 & $\mathrm{H}$ & -4.547900 & -3.861600 & -4.744871 \\
\hline $\mathrm{C}$ & -3.384269 & 0.185771 & 3.611863 & $\mathrm{H}$ & -6.303500 & 5.525005 & -2.478554 \\
\hline $\mathrm{C}$ & -3.169777 & -3.311392 & 2.683784 & $\mathrm{H}$ & -6.298247 & -0.553563 & 6.011305 \\
\hline $\mathrm{C}$ & -3.400335 & -3.236484 & -1.620009 & $\mathrm{H}$ & -6.471926 & -4.667518 & -3.445804 \\
\hline $\mathrm{C}$ & -4.472841 & 2.642524 & -2.740979 & $\mathrm{H}$ & -6.092635 & -0.269413 & -5.960614 \\
\hline $\mathrm{C}$ & -4.189701 & 4.905675 & 1.768068 & $\mathrm{H}$ & -6.055705 & 5.340267 & 2.698814 \\
\hline $\mathrm{C}$ & -4.445857 & 1.099375 & 3.652569 & $\mathrm{H}$ & -6.131506 & -4.887760 & 3.319585 \\
\hline $\mathrm{C}$ & -4.210294 & -3.933743 & 3.409787 & $\mathrm{H}$ & -1.075784 & -5.172872 & -1.939225 \\
\hline $\mathrm{C}$ & -4.457255 & -3.723563 & -0.841029 & $\mathrm{H}$ & -0.274441 & -5.632316 & -3.437207 \\
\hline $\mathrm{C}$ & -4.234178 & -0.878652 & -5.114950 & $\mathrm{H}$ & 2.283102 & -2.409755 & 6.075070 \\
\hline $\mathrm{C}$ & -4.433989 & 1.392036 & -3.469424 & $\mathrm{H}$ & 4.985373 & 0.901381 & 5.677516 \\
\hline $\mathrm{C}$ & -4.424310 & 5.190230 & -1.531180 & $\mathrm{H}$ & 3.317883 & 2.076708 & 4.246398 \\
\hline $\mathrm{C}$ & -4.397462 & 2.354040 & 2.931933 & $\mathrm{H}$ & 1.189279 & 0.965865 & 3.653799 \\
\hline $\mathrm{C}$ & -4.417560 & -1.217267 & 5.261161 & $\mathrm{H}$ & 2.697158 & -1.835121 & -5.364346 \\
\hline $\mathrm{C}$ & -4.397657 & -3.731445 & 0.607808 & $\mathrm{H}$ & 5.317651 & 0.325760 & -2.743418 \\
\hline $\mathrm{C}$ & -4.522331 & -3.815043 & -3.661688 & $\mathrm{H}$ & 3.370692 & 0.930255 & -1.308204 \\
\hline $\mathrm{C}$ & -5.480156 & 1.085430 & -4.381195 & $\mathrm{H}$ & 1.119526 & 0.033432 & -1.833596 \\
\hline $\mathrm{C}$ & -5.509587 & 4.805239 & -2.301559 & $\mathrm{H}$ & -2.042863 & -5.468459 & -3.378461 \\
\hline $\mathrm{C}$ & -5.445074 & 3.298593 & 3.107115 & $\mathrm{H}$ & 0.410147 & 0.678098 & -5.151944 \\
\hline $\mathrm{C}$ & -5.498894 & -0.352681 & 5.304179 & $\mathrm{H}$ & -1.976340 & 5.704877 & -3.048835 \\
\hline $\mathrm{C}$ & -5.445910 & -4.329583 & 1.355877 & $\mathrm{H}$ & -0.473205 & 2.663023 & 2.992145 \\
\hline $\mathrm{C}$ & -5.597535 & -4.287844 & -2.926281 & $\mathrm{H}$ & -1.958362 & -0.159649 & 6.456194 \\
\hline $\mathrm{C}$ & -5.343413 & -0.058324 & -5.203436 & $\mathrm{H}$ & 0.448736 & -4.830149 & 2.074826 \\
\hline $\mathrm{C}$ & -5.566428 & 3.533493 & -2.920342 & $\mathrm{H}$ & -7.718246 & 4.316239 & 3.182543 \\
\hline $\mathrm{C}$ & -5.304303 & 4.577512 & 2.516993 & $\mathrm{H}$ & -7.877925 & 0.487095 & 5.323788 \\
\hline $\mathrm{C}$ & -5.546396 & 0.815677 & 4.506841 & $\mathrm{H}$ & -7.504308 & -5.995414 & -2.002738 \\
\hline $\mathrm{C}$ & -5.331535 & -4.415754 & 2.761373 & $\mathrm{H}$ & -8.215640 & -5.778206 & 0.738499 \\
\hline $\mathrm{C}$ & -5.582191 & -4.280596 & -1.511820 & $\mathrm{H}$ & -7.886360 & 4.430719 & -3.051061 \\
\hline $\mathrm{C}$ & -6.679441 & 3.118825 & -3.733353 & $\mathrm{H}$ & -7.760810 & 0.662065 & -5.297531 \\
\hline $\mathrm{C}$ & -6.657861 & 1.729346 & 4.542010 & $\mathrm{Br}$ & 4.876225 & -5.228406 & -5.126395 \\
\hline $\mathrm{C}$ & -6.631667 & -4.844926 & -0.719430 & $\mathrm{Br}$ & 5.577532 & -1.336943 & -5.208498 \\
\hline $\mathrm{C}$ & -6.633819 & 1.943477 & -4.442098 & $\mathrm{Br}$ & 5.635519 & 5.148297 & 1.462115 \\
\hline $\mathrm{C}$ & -6.605058 & 2.927956 & 3.873749 & $\mathrm{Br}$ & 5.618129 & -3.928892 & 3.694327 \\
\hline $\mathrm{C}$ & -6.570684 & -4.876001 & 0.649863 & $\mathrm{Br}$ & 5.017255 & -1.778871 & 6.965113 \\
\hline $\mathrm{C}$ & -1.099908 & -5.046219 & -3.027851 & $\mathrm{Br}$ & 4.966791 & 6.995431 & -1.971174 \\
\hline \multicolumn{8}{|c|}{ HBCNR } \\
\hline $\mathrm{C}$ & 5.055784 & 3.180873 & -4.744686 & $\mathrm{C}$ & -5.041911 & 4.365900 & 2.172057 \\
\hline $\mathrm{C}$ & 5.620841 & 4.042869 & 1.027120 & $\mathrm{C}$ & -5.072594 & -4.049278 & 2.667480 \\
\hline $\mathrm{C}$ & 5.626779 & -2.900948 & 2.995901 & $\mathrm{C}$ & 0.708773 & -5.478701 & -0.216142 \\
\hline $\mathrm{C}$ & 5.062483 & -5.697977 & -0.373861 & $\mathrm{O}$ & -6.091510 & 5.182382 & 1.886540 \\
\hline $\mathrm{C}$ & 5.218840 & 1.803144 & -4.616598 & $\mathrm{O}$ & -6.194010 & 3.627803 & 4.075627 \\
\hline
\end{tabular}




\begin{tabular}{|c|c|c|c|c|c|c|c|}
\hline $\mathrm{C}$ & 5.623314 & 4.752648 & -0.171720 & $\mathrm{O}$ & -6.212011 & -5.296621 & 1.031397 \\
\hline $\mathrm{C}$ & 4.449834 & -3.011184 & 2.238848 & $\mathrm{O}$ & -6.171408 & -4.218052 & 3.467609 \\
\hline $\mathrm{C}$ & 5.225047 & -4.898912 & 0.755865 & $\mathrm{O}$ & -6.133079 & 1.799382 & -5.134070 \\
\hline $\mathrm{C}$ & 4.372057 & 1.056705 & -3.781343 & $\mathrm{O}$ & -6.140383 & -0.866770 & -5.461504 \\
\hline $\mathrm{C}$ & 4.462011 & 4.872959 & -0.935134 & $\mathrm{C}$ & 4.014494 & 1.607595 & 5.361917 \\
\hline $\mathrm{C}$ & 3.297469 & -2.386472 & 2.732509 & $\mathrm{C}$ & 5.041586 & 2.523410 & 5.131151 \\
\hline $\mathrm{C}$ & 4.377420 & -3.803220 & 0.985362 & $\mathrm{C}$ & 5.206552 & 3.104110 & 3.875497 \\
\hline $\mathrm{C}$ & 4.027183 & 3.840934 & -4.071382 & $\mathrm{C}$ & 4.363375 & 2.754367 & 2.808522 \\
\hline $\mathrm{C}$ & 4.440888 & 3.444260 & 1.496269 & $\mathrm{C}$ & 3.372996 & 1.791565 & 3.049561 \\
\hline $\mathrm{C}$ & 5.622689 & -2.218057 & 4.210230 & $\mathrm{C}$ & 3.166169 & 1.235849 & 4.314438 \\
\hline $\mathrm{C}$ & 4.033819 & -5.444949 & -1.282136 & $\mathrm{C}$ & 3.298503 & -1.168931 & -3.424433 \\
\hline $\mathrm{C}$ & 3.175509 & 3.122318 & -3.226750 & $\mathrm{C}$ & 4.447717 & -0.424711 & -3.720557 \\
\hline $\mathrm{C}$ & 3.292083 & 3.560772 & 0.703512 & $\mathrm{C}$ & 5.626855 & -1.131454 & -4.004642 \\
\hline $\mathrm{C}$ & 4.457934 & -1.620793 & 4.692480 & $\mathrm{C}$ & 5.628441 & -2.524457 & -4.019896 \\
\hline $\mathrm{C}$ & 3.181776 & -4.354570 & -1.081489 & $\mathrm{C}$ & 4.466855 & -3.245094 & -3.742262 \\
\hline $\mathrm{C}$ & 3.380499 & 1.749217 & -3.072308 & $\mathrm{C}$ & 3.283246 & -2.568144 & -3.424922 \\
\hline $\mathrm{C}$ & 3.277637 & 4.260807 & -0.508111 & $\mathrm{H}$ & 5.713926 & 3.739299 & -5.403605 \\
\hline $\mathrm{C}$ & 3.276601 & -1.687331 & 3.944391 & $\mathrm{H}$ & 6.541090 & 3.942803 & 1.593176 \\
\hline $\mathrm{C}$ & 3.386452 & -3.534753 & 0.030588 & $\mathrm{H}$ & 6.549663 & -3.338196 & 2.629494 \\
\hline $\mathrm{C}$ & 2.003447 & 4.268805 & -1.285837 & $\mathrm{H}$ & 5.721066 & -6.547390 & -0.528523 \\
\hline $\mathrm{C}$ & 1.999174 & -1.021049 & 4.334942 & $\mathrm{H}$ & 5.979442 & 1.298860 & -5.203208 \\
\hline $\mathrm{C}$ & 2.008730 & -3.244718 & -3.042611 & $\mathrm{H}$ & 6.542488 & 5.215166 & -0.518315 \\
\hline $\mathrm{C}$ & 1.962436 & 3.711326 & -2.577117 & $\mathrm{H}$ & 5.985428 & -5.154905 & 1.485953 \\
\hline $\mathrm{C}$ & 1.953968 & 0.376317 & 4.495770 & $\mathrm{H}$ & 4.475816 & 5.436676 & -1.859961 \\
\hline $\mathrm{C}$ & 1.967990 & -4.086119 & -1.915425 & $\mathrm{H}$ & 2.373305 & -2.459598 & 2.172930 \\
\hline $\mathrm{C}$ & 0.827944 & -2.127757 & -5.005786 & $\mathrm{H}$ & 3.869623 & 4.905208 & -4.216524 \\
\hline $\mathrm{C}$ & 0.732217 & 3.506944 & -3.230477 & $\mathrm{H}$ & 6.539679 & -2.146289 & 4.787222 \\
\hline $\mathrm{C}$ & 0.823481 & 5.414640 & 0.660398 & $\mathrm{H}$ & 3.876671 & -6.102260 & -2.131731 \\
\hline $\mathrm{C}$ & 0.721796 & 1.041705 & 4.640462 & $\mathrm{H}$ & 2.365819 & 3.113429 & 1.041835 \\
\hline $\mathrm{C}$ & 0.822793 & -3.282345 & 4.345218 & $\mathrm{H}$ & 4.467249 & -1.101841 & 5.643203 \\
\hline $\mathrm{C}$ & 0.737647 & -4.549585 & -1.411597 & $\mathrm{H}$ & 2.727503 & 1.211982 & -2.393798 \\
\hline $\mathrm{C}$ & 0.809560 & -2.898710 & -3.701013 & $\mathrm{H}$ & 2.733931 & -2.678149 & 0.156500 \\
\hline $\mathrm{C}$ & 0.705894 & 2.943198 & -4.635706 & $\mathrm{H}$ & 0.711453 & -1.047664 & -4.867509 \\
\hline $\mathrm{C}$ & 0.804336 & 4.668020 & -0.658458 & $\mathrm{H}$ & 0.002527 & -2.449443 & -5.645040 \\
\hline $\mathrm{C}$ & 0.691255 & 2.540840 & 4.851596 & $\mathrm{H}$ & -0.000501 & 6.130924 & 0.699684 \\
\hline $\mathrm{C}$ & 0.801836 & -1.767129 & 4.362985 & $\mathrm{H}$ & 1.764494 & 5.951964 & 0.788154 \\
\hline $\mathrm{C}$ & -0.429047 & 4.335783 & -1.255108 & $\mathrm{H}$ & 0.698998 & -3.699834 & 3.340407 \\
\hline $\mathrm{C}$ & -0.432561 & -1.086701 & 4.371372 & $\mathrm{H}$ & 0.002525 & -3.678250 & 4.948636 \\
\hline $\mathrm{C}$ & -0.423714 & -3.250514 & -3.115527 & $\mathrm{H}$ & 0.823114 & 1.853315 & -4.644254 \\
\hline $\mathrm{C}$ & -0.466872 & 3.745399 & -2.529742 & $\mathrm{H}$ & 1.526833 & 3.349428 & -5.229872 \\
\hline $\mathrm{C}$ & -0.475010 & 0.312133 & 4.494848 & $\mathrm{H}$ & 0.806598 & 3.091429 & 3.910919 \\
\hline $\mathrm{C}$ & -0.460746 & -4.062912 & -1.970169 & $\mathrm{H}$ & 1.511345 & 2.855828 & 5.499733 \\
\hline $\mathrm{C}$ & -1.733708 & -1.190689 & -3.374016 & $\mathrm{H}$ & -0.900451 & -0.721236 & -2.866197 \\
\hline $\mathrm{C}$ & -1.722647 & 3.136571 & -3.064549 & $\mathrm{H}$ & -0.909570 & 2.844221 & 0.798930 \\
\hline $\mathrm{C}$ & -1.738246 & 3.525903 & 0.654202 & $\mathrm{H}$ & -0.927175 & -2.102850 & 2.045427 \\
\hline $\mathrm{C}$ & -1.733094 & 1.074001 & 4.233018 & $\mathrm{H}$ & -0.904574 & 1.214928 & -2.720313 \\
\hline $\mathrm{C}$ & -1.750924 & -2.325370 & 2.711711 & $\mathrm{H}$ & -0.913147 & 1.751841 & 2.402646 \\
\hline $\mathrm{C}$ & -1.718394 & -4.225571 & -1.180080 & $\mathrm{H}$ & -0.926280 & -2.942012 & 0.303105 \\
\hline $\mathrm{C}$ & -1.659293 & -2.559570 & -3.592321 & $\mathrm{H}$ & -2.680079 & 6.009700 & -1.448899 \\
\hline
\end{tabular}




\begin{tabular}{|c|c|c|c|c|c|c|c|}
\hline $\mathrm{C}$ & -1.762495 & 1.750167 & -3.106950 & $\mathrm{H}$ & -2.655139 & -1.818139 & 5.933577 \\
\hline $\mathrm{C}$ & -1.663424 & 4.405629 & -0.415715 & $\mathrm{H}$ & -2.675198 & -4.254124 & -4.468600 \\
\hline $\mathrm{C}$ & -1.774263 & 1.810264 & 3.056432 & $\mathrm{H}$ & -2.805860 & 4.942489 & -3.556640 \\
\hline $\mathrm{C}$ & -1.665488 & -1.850095 & 4.012034 & $\mathrm{H}$ & -2.811908 & 0.606155 & 6.047070 \\
\hline $\mathrm{C}$ & -1.776319 & -3.555944 & 0.033683 & $\mathrm{H}$ & -2.764163 & -5.614876 & -2.464589 \\
\hline $\mathrm{C}$ & -2.839631 & 1.037269 & -3.660028 & $\mathrm{H}$ & -4.769545 & 3.745233 & -4.470585 \\
\hline $\mathrm{C}$ & -2.732679 & 5.299460 & -0.631142 & $\mathrm{H}$ & -4.752380 & 2.013914 & 5.495359 \\
\hline $\mathrm{C}$ & -2.850026 & 2.645015 & 2.715908 & $\mathrm{H}$ & -4.695373 & -5.852119 & -0.983376 \\
\hline $\mathrm{C}$ & -2.720325 & -2.139984 & 4.899821 & $\mathrm{H}$ & -4.633317 & -2.955809 & -5.202956 \\
\hline $\mathrm{C}$ & -2.854330 & -3.681786 & 0.924885 & $\mathrm{H}$ & -4.664668 & 5.962871 & 0.032129 \\
\hline $\mathrm{C}$ & -2.729144 & -3.191693 & -4.256738 & $\mathrm{H}$ & -4.599470 & -3.102018 & 5.185459 \\
\hline $\mathrm{C}$ & -2.820497 & -0.409212 & -3.797735 & $\mathrm{H}$ & 0.814557 & -4.934125 & 0.729317 \\
\hline $\mathrm{C}$ & -2.825938 & 3.858632 & -3.564629 & $\mathrm{H}$ & 1.534496 & -6.191412 & -0.259284 \\
\hline $\mathrm{C}$ & -2.826676 & 3.502245 & 1.542981 & $\mathrm{H}$ & 3.855202 & 1.199252 & 6.355138 \\
\hline $\mathrm{C}$ & -2.835604 & 1.146975 & 5.107418 & $\mathrm{H}$ & 5.696933 & 2.814902 & 5.946450 \\
\hline $\mathrm{C}$ & -2.836745 & -3.085682 & 2.247066 & $\mathrm{H}$ & 5.965703 & 3.866251 & 3.735573 \\
\hline $\mathrm{C}$ & -2.802944 & -5.049237 & -1.540235 & $\mathrm{H}$ & 2.722541 & 1.473138 & 2.242587 \\
\hline $\mathrm{C}$ & -3.908874 & -1.067989 & -4.438345 & $\mathrm{H}$ & 2.372393 & -0.651931 & -3.206502 \\
\hline $\mathrm{C}$ & -3.920356 & 3.193013 & -4.085616 & $\mathrm{H}$ & 6.547312 & -0.591970 & -4.201900 \\
\hline $\mathrm{C}$ & -3.905373 & 4.394603 & 1.294916 & $\mathrm{H}$ & 6.547094 & -3.056380 & -4.248122 \\
\hline $\mathrm{C}$ & -3.928746 & 1.934839 & 4.792763 & $\mathrm{H}$ & 4.479930 & -4.327848 & -3.768623 \\
\hline $\mathrm{C}$ & -3.918061 & -3.331567 & 3.138909 & $\mathrm{H}$ & -0.231268 & -6.029519 & -0.169590 \\
\hline $\mathrm{C}$ & -3.896945 & -5.171336 & -0.702469 & $\mathrm{H}$ & 1.767942 & -2.285906 & -5.536798 \\
\hline $\mathrm{C}$ & -3.831914 & -2.461592 & -4.662794 & $\mathrm{H}$ & -0.237292 & 3.172435 & -5.132575 \\
\hline $\mathrm{C}$ & -3.944752 & 1.783260 & -4.153798 & $\mathrm{H}$ & 0.705172 & 4.756662 & 1.527722 \\
\hline $\mathrm{C}$ & -3.837656 & 5.283072 & 0.199981 & $\mathrm{H}$ & -0.253024 & 2.854854 & 5.297624 \\
\hline $\mathrm{C}$ & -3.963619 & 2.696241 & 3.602284 & $\mathrm{H}$ & 1.766107 & -3.662848 & 4.739706 \\
\hline $\mathrm{C}$ & -3.821387 & -2.857260 & 4.468127 & $\mathrm{H}$ & -6.753238 & 5.023681 & 2.579326 \\
\hline $\mathrm{C}$ & -3.957610 & -4.492678 & 0.537250 & $\mathrm{H}$ & -6.502604 & 2.731663 & 4.267764 \\
\hline $\mathrm{C}$ & -5.058390 & 1.075883 & -4.720272 & $\mathrm{H}$ & -6.274117 & -5.265021 & 0.068175 \\
\hline $\mathrm{C}$ & -5.065668 & 3.545114 & 3.267687 & $\mathrm{H}$ & -6.211283 & -3.479028 & 4.088008 \\
\hline $\mathrm{C}$ & -5.093343 & -4.604853 & 1.413541 & $\mathrm{H}$ & -6.771827 & 1.157389 & -5.484611 \\
\hline $\mathrm{C}$ & -5.033655 & -0.286636 & -4.852266 & $\mathrm{H}$ & -6.423577 & -1.624006 & -4.930826 \\
\hline
\end{tabular}




\section{References.}

(S1) Z. He, X. Xu, X. Zheng, T. Ming and Q. Miao, Chem. Sci., 2013, 4, 4525-4531.

(S2) X. Dou, T. Hayashi, Adv. Synth. Catal., 2016, 358, 1054-1058.

(S3) F. Romanov-Michailidis, C. Besnard, A. Alexakis, Org. Lett., 2013, 44(5), 4906-4909.

(S4) J. Tirado-Rives, W. L. Jorgensen, J. Chem. Theory Comput., 2008, 4 (2), 297306.

(S5) T. Yanai, D. P. Tew, N. C. Hany, Chem. Phys. Lett., 2004, 393, 51.

(S6) B. Menucci, J. Tomasi, J. Phys. Chem. A., 2002, 106, 6102.

(S7) Y. Segawa, H. Omachi, K. Itami, Org. Lett., 2010, 12, 2262.

(S8) H.Takaba, H.Omachi, Y.Yamamoto, J. Bouffard, K. Itami, Angew. Chem., Int. Ed., 2009, 48, 6112. 